\title{
Permanent Magnet Synchronous Generator for Wind Turbines: Modelling, Control and Inertial Frequency Response
}

\author{
Marc Cheah-Mane \\ Cardiff University, UK \\ CheahM@cf.ac.uk
}

\author{
Jun Liang \\ Cardiff University, UK \\ LiangJ1@cf.ac.uk
}

\author{
Nick Jenkins \\ Cardiff University, UK \\ JenkinsN6@cf.ac.uk
}

\begin{abstract}
Offshore wind power generation is expected to increase in the following years, but there are still some economic and technical challenges to overcome. Because of the difficult access to the offshore facilities, the reduction of maintenance is an essential point. The use of Permanent Magnet Synchronous Generators (PMSG) is considered a suitable option in this wind farm topology to satisfy this purpose. On the other hand, these generators along with full-rated Voltage Source Converters (VSC) are expected to provide ancillary services for the onshore AC grid. Particularly, Inertial Frequency Response is an interesting option considering the stored kinetic energy in the rotor of the wind turbines. Therefore, in this paper a description of the model and control system of a PMSG using full-rated VSC are reviewed and their Inertial Frequency Response capability is presented through two methodologies. At the end, simulation and experimental tests results are shown in order to compare these two different options and analyse their viability.
\end{abstract}

Index Terms-Experimental Platform, Frequency Support, Offshore Wind Turbines, Permanent Magnet Synchronous Generator, Voltage Source Converter.

\section{INTRODUCTION}

The wind power generation is one of the most promising options in the contribution to the increase of renewal energies. In particular, there is a recent interest in offshore wind power because of the lack of onshore space in some areas and the possibility to set up facilities with higher power rates.

However, despite these advantages, there are some economic and technical challenges to overcome. In particular, the decrease of maintenance and the increase of reliability, comparing to the conventional onshore wind farms, are essential aspects considering the remote location of the offshore facilities. From this point of view, Permanent Magnet Synchronous Generator (PMSG) presents several advantages to be the most suitable solution among the different types of generators [1], especially for high rated power wind turbines. The gear box, which is the component that causes more failures in a wind turbine, can be removed with a multi-pole configuration of the stator. Moreover, the electrical excitation is replaced by permanent magnets which eliminates the losses related to the rotor winding and reduces the weight of the generator.

On the other hand, the Offshore Wind Farms (OWF) should provide auxiliary support to the onshore AC main grid [2], [3]. One of these requirements is the frequency support to avoid an excessive deviation in relation to the nominal value.
The grid code of each country has to consider this issue and provide specific limits and restoration procedures. For example, in UK when there is a sudden change of generation or load, the frequency can deviate temporally until $\pm 0.5 \mathrm{~Hz}$ [4].

PMSG has to be controlled by a full-rated Voltage Source Converter (VSC) that provides a variable speed operation for the wind turbine, but at the same time it becomes insensible to the frequency changes in the main grid. However, additional control strategies can be applied to provide the necessary support. Several studies has been already done with Doubly-Fed Induction Generators (DFIG) to prove the feasibility of different solutions [5], [6] and a detailed analysis of the factors that can affect inertia response is presented in [7]. The same methodologies can be also applied for PMSG as is shown in [8].

Therefore, in this paper a PMSG dynamic model and control configuration are presented to extract the maximum power from the wind in normal operation and to provide frequency support in case of a sudden change of load or generation. Concretely, the inertial frequency response of the wind turbines is analysed to reduce the Rate of Change of Frequency (RoCoF) during the critical first seconds of a low frequency event. Two different methodologies are presented and compared to create synthetic inertia and provide the necessary frequency support.

On the other hand, the previous studies about inertial response only have shown results through different simulations tools. Hence, another objective of this paper is to implement several control strategies in a scaled down experimental platform to verify the feasibility of this solution.

\section{Permanent Magnet Synchronous Generator MODEL}

The electrical machine models are based on the voltage and flux equations. Specifically, PMSG does not have equations associated to the rotor windings because the excitation is produced by permanent magnets. Therefore, is possible to define:

$$
\begin{gathered}
v_{s}^{a b c}=r_{s} i_{s}^{a b c}+\frac{d}{d t} \lambda_{s}^{a b c} \\
\lambda_{s}^{a b c}=L_{s} i_{s}^{a b c}+\lambda_{m}
\end{gathered}
$$


Where, $v_{s}^{a b c}$ and $i_{s}^{a b c}$ are the voltages and currents in the stator windings, $r_{s}$ and $L_{s}$ are the resistance and inductance associated with the stator windings and $\lambda_{s}^{a b c}$ is the magnetic flux in the stator. It can be observed that the magnetic flux in the stator is generated by the inductance associated to the stator and the magnetic flux of the permanent magnet, $\lambda_{m}$, which is considered a constant value.

The dynamic response of the electrical machines is usually analysed using a dq frame considering the rotor speed, $\omega_{r}$, as a reference for the Park transformation:

$$
\begin{gathered}
\left\{\begin{array}{c}
v_{q s}=r_{s} i_{q s}+\frac{d \lambda_{q s}}{d t}+\lambda_{q s} \omega_{r} \\
v_{d s}=r_{s} i_{d s}+\frac{d \lambda_{d s}}{d t}+\lambda_{d s} \omega_{r}
\end{array}\right. \\
\left\{\begin{array}{c}
\lambda_{q s}=L_{q} i_{q s} \\
\lambda_{d s}=L_{d} i_{d s}+\lambda_{m}
\end{array}\right.
\end{gathered}
$$

Where, $L_{q}$ and $L_{d}$ are the inductances associated with $\mathrm{d}$ and $\mathrm{q}$ axis. The elements related to the homopolar axis have been neglected considering that there is always a symmetric and balanced three-phase system.

Moreover, from (3) and (4) it is possible to define voltagecurrent and torque equations:

$$
\begin{aligned}
& \left\{\begin{array}{c}
v_{q s}=r_{s} i_{q s}+L_{q} \frac{d i_{q s}}{d t}+\omega_{r} L_{d} i_{d s}+\omega_{r} \lambda_{m} \\
v_{d s}=r_{s} i_{d s}+L_{d} \frac{d i_{d s}}{d t}+\omega_{r} L_{q} i_{q s}
\end{array}\right. \\
& T_{m}=\frac{3}{2} p\left(\lambda_{m} i_{q s}+\left(L_{d}-L_{q}\right) i_{q s} i_{s d}\right)
\end{aligned}
$$

Where, $p$ is the number of pole pairs. It is observed that whether $i_{s d}$ zero or the machine has surface mounted magnets $\left(L_{d}=L_{q}\right)$, the torque will have a direct relationship with $i_{q s}$. More details about the modelling can be found in [9].

\section{WIND TURBINE CONTROL}

Fig. 1 shows the general control scheme for an offshore wind turbine based on PMSG with full-rated VSC. It can be observed that there are 3 different control blocks: (i) Optimum Wind Power extraction; (ii) Generator Control; and (iii) DC link Voltage Control.

The Optimum Wind Power extraction is not applied over all wind speeds because of power converter and mechanical limitations. Therefore, for very low wind speed values the rotor speed is kept almost constant and for high values there is a limit related to the nominal rotor speed and nominal

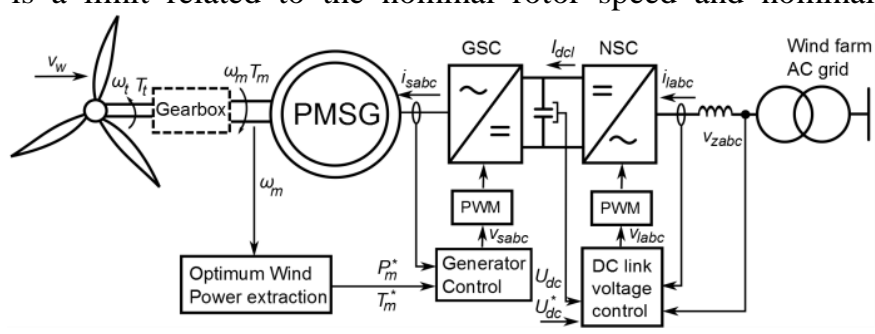

Fig. 1. General control scheme of an OWF based on PMSG with full-rated VSC. power. Above these nominal values the power or torque reference is saturated through aerodynamic procedures, such as pitch angle control.

The full-rated converter is based on back-to-back Voltage Source Converters (VSC) that provides a variable speed operation for the PMSG. In particular, the Generator Side Converter (GSC) controls the power extracted from the wind turbine, whereas the Network Side Converter (NSC) is responsible for keeping constant the voltage of the DC link between the two VSC.

\section{A. Optimum Wind Power extraction}

The Optimum Wind Power extraction provides the necessary power or torque reference based on the operational points of maximum efficiency. This optimal reference value depends on the wind speed, rotor speed and pitch angle and is related to the maximization of the power coefficient, $C_{p}$, which represents the aerodynamic efficiency. Therefore, it is possible to define [10]:

$$
P_{m}^{*}=P_{o p t}=K_{o p t} \omega_{r}^{3}
$$

Where, $K_{o p t}$ is the constant to optimize the power. This value can be provided by the manufacturer or can be obtained considering an analytic expression of $C_{p}[11]$.

\section{B. Generator Control}

The GSC is responsible for controlling the mechanical torque or power transmitted by the PMSG. There are basically two different types of control strategies [2]: (i) load angle control; and (ii) vector control.

The vector control is based on dq components and provides a more accurate dynamic response, hence it will be the selected control strategy in this paper.

Fig 2. shows the control scheme considering torque as a reference input. A current control has been implemented through PI controllers. The torque is directly related to $i_{s q}$, considering $L_{d}=L_{q}$, and $i_{s d}$ can be used for other purposes or simply kept zero.

Although there is an independent controller for each axis, it is easy to observe in (5) that the two components are coupled. To solve this problem a possibility consists of designing the controllers considering only the decoupled elements of the model and later include the coupled terms. However, this solution requires having an accurate value of the model parameters to ensure that the addition of the coupled elements can improve the control response.

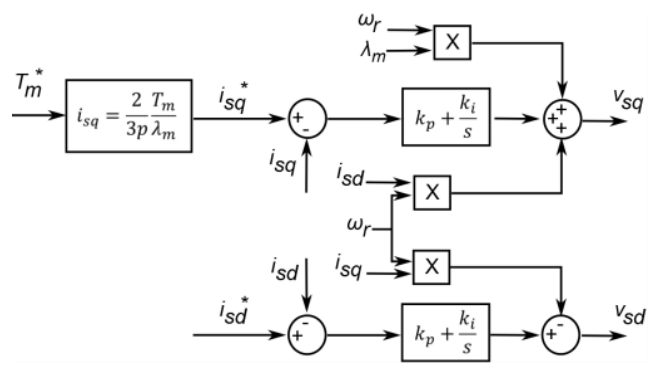

Fig. 2. General control scheme of an OWF based on PMSG with full-rated VSC. 


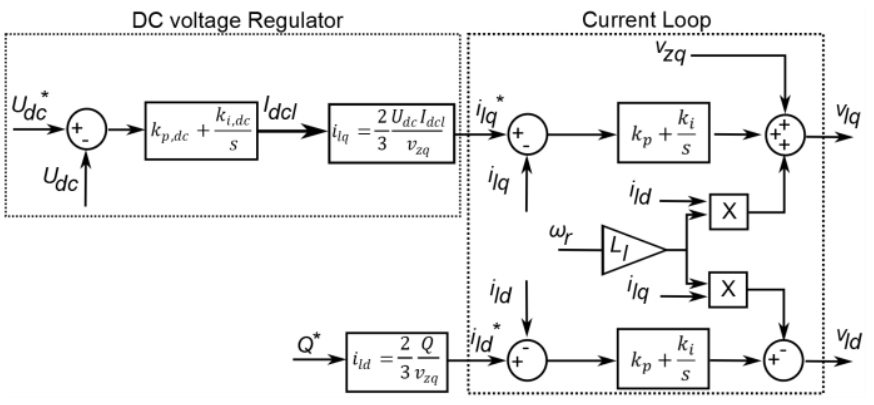

Fig. 3. DC link Voltage Control scheme

The outputs of this control system are the voltages that have to be modulated by GSC to obtain the desirable currents.

\section{DC link Voltage Control}

The NSC is responsible for keeping the DC link voltage constant and it is able to provide reactive power.

Fig. 3 shows the control scheme which has an outer loop that regulates the DC voltage through a PI controller and an inner loop that controls the current. As in the generator control, the same control technique has been selected for the current loop. However, in that case the q component is linked to the outer loop through the power transferred between the $\mathrm{AC}$ and DC side of the NSC and the d component is related to the reactive power.

The outputs of this control block are, as in the previous case, the voltages that will be modulated by NSC.

\section{INERTIAL FREQUENCY RESPONSE}

The power that can provide an electrical machine coupled directly to the grid through its inertia can be express as:

$$
\Delta P_{m}=J \omega_{r} \frac{d \omega_{r}}{d t}
$$

Where, $J$ is the inertia constant. Therefore, the kinetic energy released by the machine when the rotor speed changes from $\omega_{r 0}$ to $\omega_{r 1}$ is:

$$
\Delta E_{k}=\frac{1}{2} J \omega_{r 0}\left(1-\frac{\omega_{r 1}^{2}}{\omega_{r 0}^{2}}\right)
$$

To use the inertia response of the PMSG it is necessary to modify the control system of the generator. The power or torque reference has to include an additional term to emulate the inertia response when a frequency variation is detected.

Two different methodologies are considered by different authors:

- Inertial coupling [6], [7], [12].

- Step response [7], [13], [14].

\section{A. Inertial coupling}

This technique consists of increasing the torque or the power reference considering the derivative term of the frequency evolution.

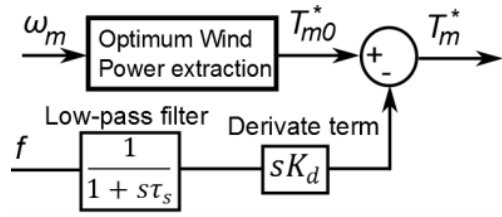

Fig. 4. Inertial coupling scheme with torque as a reference
Therefore, the electrical machine can have a response similar to the natural inertia. Fig. 4 depicts the additional control loop in case to use a torque reference where $K_{d}$ can define the synthetic inertia constant. Some authors include a low-pass filter to reduce the noise of the frequency measurement and to avoid possible instabilities caused by the derivative term [7], [12].

The advantage of this control is that the electrical machine can provide even more frequency support if it is considered an artificial inertia higher than the natural response. However, when the electrical machine releases this additional energy the rotor speed decreases causing mainly two problems. Firstly, if the rotor speed is reduced too much the wind turbine can become unstable [8]. Secondly, the rotor speed has to come back to the original value and a recovery period is necessary. Therefore, the electrical machine has to absorb temporarily a certain amount of power causing a reduction of power generation. This latter problem is not possible to avoid, except if the wind turbine operates at rated power and the pitch angle is reduced to provide additional power [6], [12].

Other solutions can be applied when the wind turbine is below the nominal conditions, for example de-loaded operation or droop control [6], [12]. However, they do not provide a real solution because the wind turbines have to operate for a long period of time below optimal power extraction.

\section{B. Step response}

This other methodology applies a constant torque or power for a specific period of time. This time can be predefined or can be related to other variables, for example the time to reach the minimum rotor speed allowed to avoid instability. Fig. 5 shows a possible control scheme where torque is the reference. It can be observed that when a frequency variation is detected the comparator enables a step change of constant torque, $T_{\text {step }}^{*}$, and after a period of time, $\Delta t$, it is automatically disabled. The authors of [14] use a similar method with power as a reference where they also control the power during the recovery period.

The main advantage of this technique is that there is not any derivative term, avoiding all the associated stability problems. However, this option can cause a high mechanical stress on the shaft when the constant torque is enabled and disabled. Moreover, as in the previous case, the recovery energy problem cannot be solved.

\section{EXPERIMENTAL PLATFORM DESCRIPTION}

A test rig has been used to obtain the necessary results. The platform consists of two coupled permanent magnet machines: one is working as a generator and represents the real PMSG and the other as a motor that emulates the wind turbine transmitting the necessary power to the generator. The two machines are connected to the grid through power

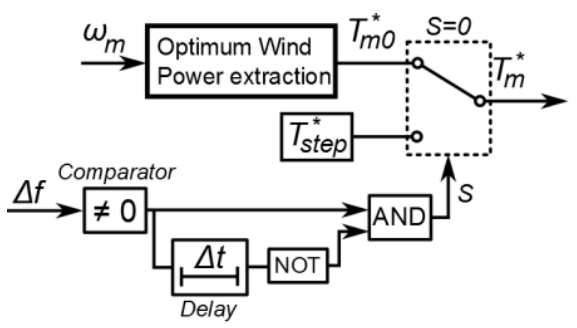

Fig. 5. Step control scheme with torque as a reference 
electronic converters, namely, a back-to-back based on Voltage Source Converters (VSC) for the generator and a variable speed motor drive for the motor. The power absorbed from the grid is returned, therefore, the total system only consumes the power related to the losses of each element. A scheme of the system is depicted in Fig. 6 and their main characteristics are specified in Table I.

TABLE I

PARAMETERS EXPERIMENTAL PLATFORM

\begin{tabular}{|c|c|}
\hline \multicolumn{2}{|c|}{ Permanent magnet machines } \\
\hline Nominal power & $1.2 \mathrm{kVA}$ \\
\hline Nominal voltage (L-L rms) & $400 \mathrm{~V}$ \\
\hline Nominal torque & $3.9 \mathrm{Nm}$ \\
\hline Nominal speed & $3000 \mathrm{rpm}$ \\
\hline Pole number & 6 \\
\hline \multicolumn{2}{|c|}{ Voltage Source Converters } \\
\hline Topology & $\begin{array}{c}\text { Two-level, three-phase without } \\
\text { neutral wire, IGBT switcher }\end{array}$ \\
\hline Nominal Power & $10 \mathrm{kVA}$ \\
\hline Nominal AC voltage (L-L rms) & $400 \mathrm{~V}$ \\
\hline Nominal DC voltage & $800 \mathrm{~V}$ \\
\hline Switching frequency & $4 \mathrm{kHz}$ \\
\hline
\end{tabular}

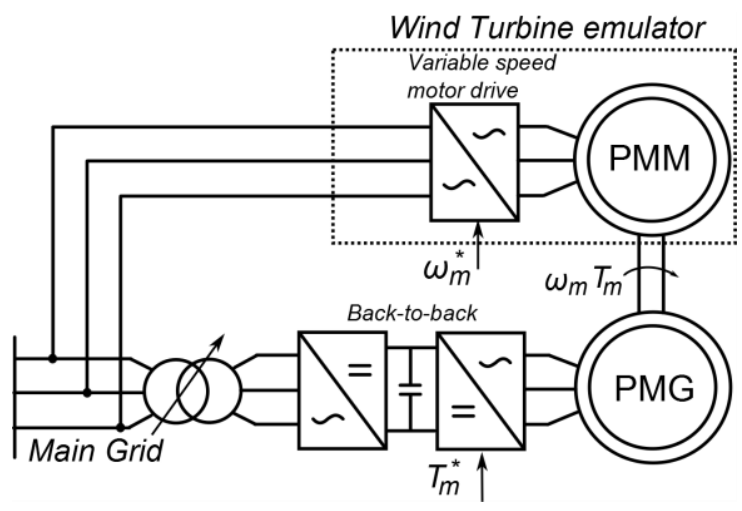

Fig. 6. Experimental platform scheme

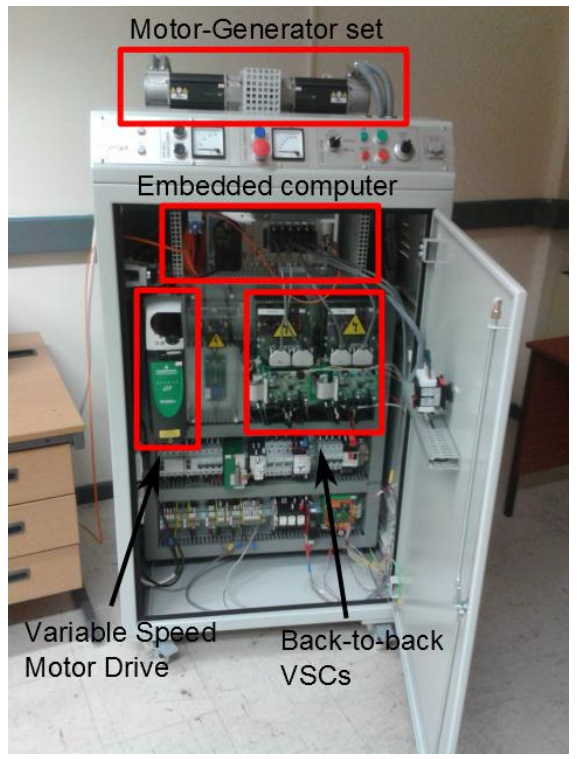

Fig. 7. Experimental platform picture

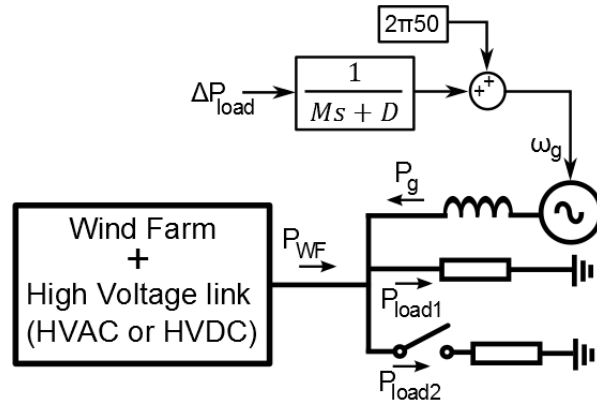

Fig. 8. AC main grid model for frequency response analysis

The converters of each machine can provide an entire control over the mechanical torque and speed of the coupling shaft. Specifically, one of the machines has to control speed and the other torque. The back-to-back control system can be easily reconfigured for any purpose through an embedded computer, whereas it is only possible to send a reference value (torque or speed) for the variable speed motor drive.

The selected configuration of the test rig is based on the control strategy described in the previous sections. Hence, the back-to-back along with the generator controls torque and the variable speed motor drive along with the motor controls speed. The aerodynamic response of the wind turbine is modelled and only the rotor speed reference is sent to the motor.

\section{CASe STUdy}

In order to evaluate the frequency support an aggregated model of an OWF is used. Moreover, it is supposed that the wind farm will be directly connected to the onshore grid through a HVAC link or through a HVDC point to point link that is able to transmit the change of frequency to the $\mathrm{AC}$ offshore grid [15]. Hence, the full-rated VSC will be able to detect the drop of frequency locally.

To analyse the effect of the additional power released by the PMSG the AC main grid has been represented as a dynamic model based on a first order response with inertia and damping constants [16]:

$$
\frac{\Delta \omega_{g}}{\Delta P_{\text {load }}}=\frac{1}{M s+D}=\frac{K_{g}}{1+s T_{g}}
$$

Where, $M$ is the inertia constant and $D$ is the load-damping constant. Fig. 8 shows the electrical scheme of the AC main grid model, where it can be observed that initially there is a consumption represented as an active power load, $P_{\text {load } 1}$. A new consumption, $P_{\text {load } 2}$, is suddenly connected to create an unbalanced condition and cause a reduction of the grid frequency. On the other hand, it is supposed that only the OWF is going to provide frequency support to the onshore grid.

The characteristics of the case study are specified in Table II.

\section{RESULTS}

When the additional load is connected and the frequency begins to drop the two different methodologies explained in 
TABLE II

CHARACTERISTICS OF THE CASE STUDY

\begin{tabular}{|c|c|}
\hline \multicolumn{2}{|c|}{ Wind turbines (PMSG + VSC) } \\
\hline Nominal power & $5 \mathrm{MVA}$ \\
\hline Nominal voltage (L-L rms) & $0.6 \mathrm{kV}$ \\
\hline Nominal frequency PMSG & $30 \mathrm{~Hz}$ \\
\hline Pole number PMSG & 250 \\
\hline Nominal DC voltage VSC & $1.2 \mathrm{kV}$ \\
\hline Switching frequency VSC & $1.64 \mathrm{kHz}$ \\
\hline Inertia constant & $10^{6} \mathrm{~kg} \cdot \mathrm{m}^{2}$ \\
\hline \multicolumn{2}{|c|}{ Wind farm } \\
\hline Nominal voltage (L-L rms) & $33 \mathrm{kV}$ \\
\hline Number of wind turbines & 100 \\
\hline Connection topology & $\mathrm{Radial}$ \\
\hline \multicolumn{2}{|c|}{ AC main grid } \\
\hline$P_{\text {load } 1}$ & $600 \mathrm{MW}$ \\
\hline$P_{\text {load } 2}$ & $300 \mathrm{MW}$ \\
\hline$K_{g}$ & $0.01 \mathrm{rad} /(\mathrm{s} \cdot \mathrm{MW})$ \\
\hline$T_{g}$ & $0.1 \mathrm{~s}$ \\
\hline
\end{tabular}

section IV, using torque as a reference, will be analysed and compared in relation to their capability to reduce the RoCoF. The results have been obtained using PSCAD/EMTDC software tool and the experimental platform presented in section $\mathrm{V}$. In both cases the response of the grid frequency is based on the AC grid model described in section VI. On the other hand, it is supposed that all the wind turbines are operating at constant medium wind speed $(9 \mathrm{~m} / \mathrm{s}$ which corresponds to a $40 \%$ of their rated power).

Fig. 9 shows the results applying the inertial coupling solution. For all the cases the low pass filter has $\tau_{s}=0.001 \mathrm{~s}$. It is clear that increasing $K_{d}$ the additional torque is higher and the RoCoF is reduced. However, at the same time the drop of rotor speed is also higher and more recovery energy is required. As a consequence, the additional torque during the recovery period is lower and that represents a negative effect over the RoFoC and the minimum frequency. To avoid this excessive drop of frequency other types of frequency support techniques should be applied.

In the test results it can be observed that the additional torque shows undesirable oscillations, especially when $K_{d}=0.2$, because of the derivative term.

Fig. 10 shows the results for the step response solution. It is clear that the frequency presents two different responses which are related to the constant torque and the recovery period. When the constant torque is applied the RoCoF is improved, but once the constant torque is disabled and the recovery starts the frequency drops more rapidly and the minimum value of frequency is inferior compared to the case without step control. Moreover, either if the same constant torque is kept for a longer time or the value of this torque is higher for the same time, the recovery energy is also larger.
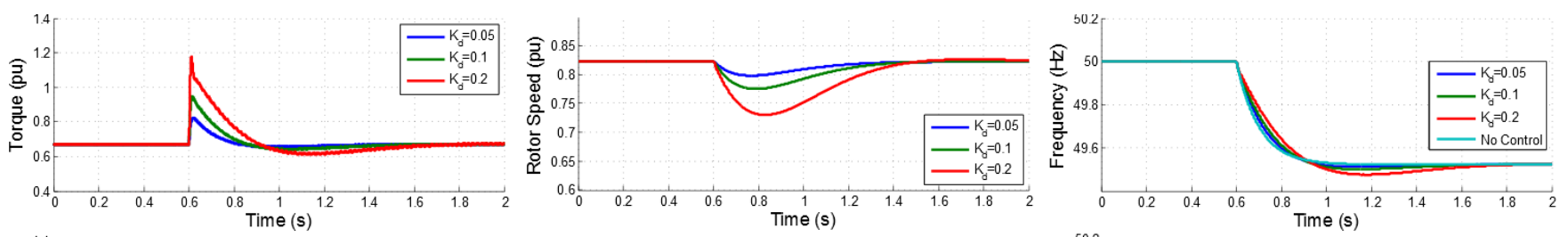

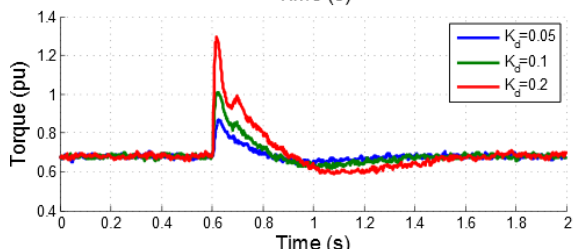

(a) Mechanical torque

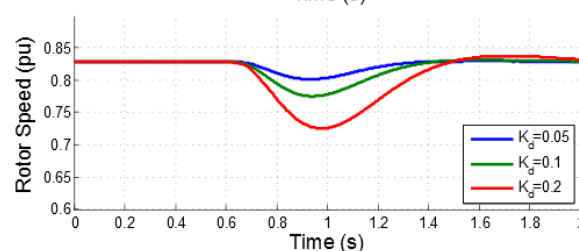

(b) Rotor speed of each PMSG

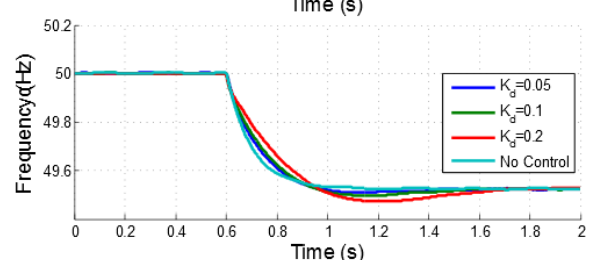

(c) Grid frequency

Fig. 9. Results for inertial coupling method with torque as a reference. Above simulation results, below test results.
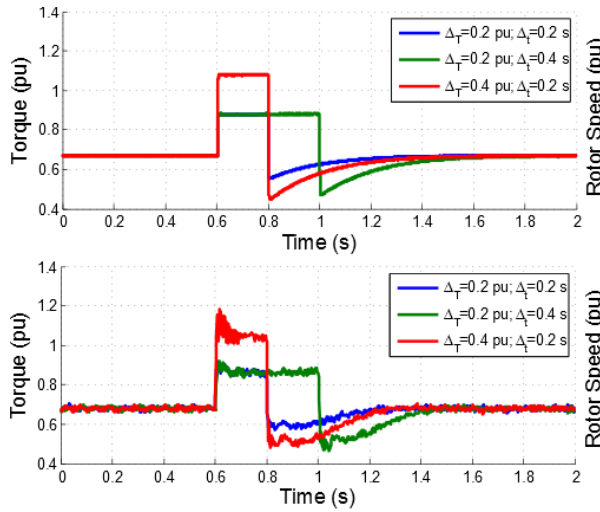

(b) Mechanical torque
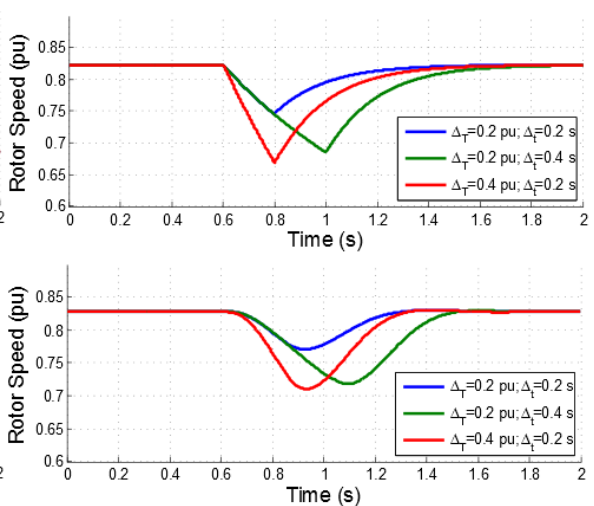

(b) Rotor speed of each PMSG
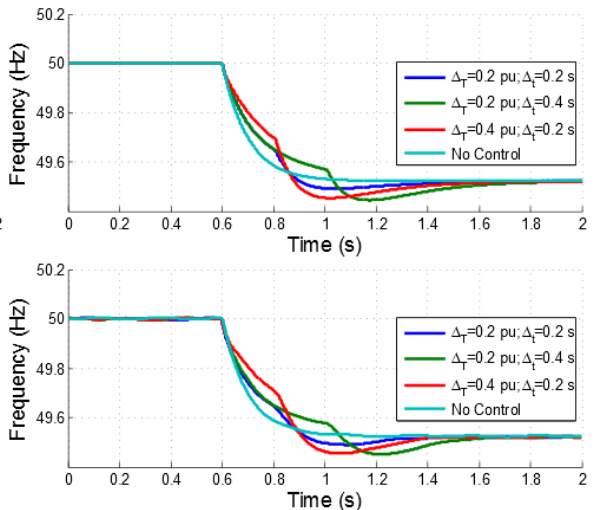

(c) Grid frequency

Fig. 10. Results for step response method with torque as a reference. Above simulation results, below test results. 
In the test results it is also possible to observe undesirable oscillations when the additional torque is enabled or disabled because of the step change of reference value.

On the other hand, good agreement can be observed between simulation and test results for both methodologies, proving the capabilities of the experimental platform to perform these studies.

\section{CONCLUSIONS}

This paper has reviewed the modelling and control system of a PMSG for OWF applications. The inertial frequency response of variable speed wind turbines has been explained making emphasis at two main methodologies: inertial coupling and step response.

An experimental platform to analyse wind turbine topologies based on full-rated converters and PMSG has been described and different tests have been performed for frequency response to verify their capabilities.

The simulation and experimental results have good agreement and demonstrate the possibility to reduce the RoCoF, but at the same time they show the recovery energy problem to restore the initial rotor speed. Moreover, comparing the two methodologies, it has been observed that the inertial coupling technique provides a synthetic inertia with the same dynamic response as the natural inertia, but the addition of a derivative term can destabilise the system. On the other hand, the step response technique has the advantage to avoid the derivate term, but it can cause mechanical stress in the shaft when the additional torque is enabled or disabled.

It is expected that this experimental platform will be used along with Real Time Digital Simulators to represent more detailed models of the AC main grid or along with other platforms to perform more complex studies.

\section{ACKNOWLEDGEMENTS}

The research leading to these results has received funding from the People Programme (Marie Curie Actions) of the European Union's Seventh Framework Programme FP7/2007-2013/ under REA grant agreement no. 317221, project title MEDOW.

\section{REFERENCES}

[1] V. Akhmatov and A. Nielsen, "Variable-speed wind turbines with multi-pole synchronous permanent magnet generators. Part I: Modelling in dynamic simulation tools.,"Wind Eng., vol. 27, pp. 531548, 2003.

[2] O. Anaya-Lara, N. Jenkins, J. Ekanayake, P. Cartwright, and M. Hughes, Wind Energy Generation: Modelling and Control. John Wiley \& Sons, 2011, p. 288.

[3] R. W. and D. M. B. Fox, D. Flynn L. Bryans, N. Jenkins, M. O' Malley, Wind Power Integration: Connection and System Operational Aspects. 2007, p. 276.

[4] I. A. Erinmez, D. O. Bickers, G. F. Wood, and W. W. Hung, "NGC experience with frequency control in England and Wales-provision of frequency response by generators," in IEEE Power Engineering Society. 1999 Winter Meeting (Cat. No.99CH36233), 1999, vol. 1, pp. 590-596 vol.1.

[5] L. Holdsworth, J. B. Ekanayake, and N. Jenkins, "Power system frequency response from fixed speed and doubly fed induction generator-based wind turbines," Wind Energy, vol. 7, no. 1, pp. 21-35, Jan. 2004.

[6] G. Ramtharan, N. Jenkins, and J. Ekanayake, "Frequency support from doubly fed induction generator wind turbines," IET Renew. Power Gener., pp. 3-9, 2007.

[7] M. Kayikci and J. V. Milanovic, "Dynamic Contribution of DFIGBased Wind Plants to System Frequency Disturbances," IEEE Trans. Power Syst., vol. 24, no. 2, pp. 859-867, May 2009.

[8] Z. Wu, W. Gao, J. Wang, and S. Gu, "A coordinated primary frequency regulation from Permanent Magnet Synchronous Wind Turbine Generation," in 2012 IEEE Power Electronics and Machines in Wind Applications, 2012, pp. 1-6.

[9] P. C. Krause, O. Wasynczuk, S. D. Sudhoff, and I. P. E. Society, Analysis of electric machinery and drive systems. IEEE Press, 2002.

[10] L. Holdsworth, X. Wu, J. Ekanayake, and N. Jenkins, "Comparison of fixed speed and doubly-fed induction wind turbines during power system disturbances," IEE Proceedings-Gener. Transm. Distrib., vol. 150, no. 3, pp. 343-352, 2003.

[11] A. Junyent Ferré, "Control of power electronic converters for the operation of wind generation," Universitat Politècnica de Catalunya, 2011.

[12] J. F. Conroy and R. Watson, "Frequency Response Capability of Full Converter Wind Turbine Generators in Comparison to Conventional Generation," IEEE Trans. Power Syst., vol. 23, no. 2, pp. 649-656, May 2008.

[13] G. C. Tarnowski, P. C. Kjar, P. E. Sorensen, and J. Ostergaard, "Variable speed wind turbines capability for temporary overproduction," in 2009 IEEE Power \& Energy Society General Meeting, 2009, pp. $1-7$.

[14] N. R. Ullah, T. Thiringer, and D. Karlsson, "Temporary Primary Frequency Control Support by Variable Speed Wind TurbinesPotential and Applications," IEEE Trans. Power Syst., vol. 23, no. 2, pp. 601-612, May 2008

[15] Y. Li, Z. Zhang, Y. Yang, Y. Li, H. Chen, and Z. Xu, "Coordinated control of wind farm and VSC-HVDC system using capacitor energy and kinetic energy to improve inertia level of power systems," Int. J. Electr. Power Energy Syst., vol. 59, pp. 79-92, Jul. 2014.

[16] P. Kundur, Power System Stability and Control. McGraw-Hill Education, 1994, p. 1176. 